ISSN 2078-3744. Вісник Лъвів. ун-ту. Серія мех.-мат. 2019. Випуск 88. С. 5-11

Visnyk of the Lviv Univ. Series Mech. Math. 2019. Issue 88. P. 5-11

http://publications.lnu.edu.ua/bulletins/index.php/mmf

doi: http://dx.doi.org/10.30970/vmm.2019.88.005-011

УДК 511.3

\title{
SIMULTANEOUS APPROXIMATION OF VALUES OF JACOBI ELLIPTIC FUNCTIONS IN THEIR REAL PERIODS
}

\author{
Yaroslav KHOLYAVKA, Olga MYLYO \\ Ivan Franko National University of Lviv, \\ Universytetska Str., 1, 79000, Lviv, Ukraine \\ e-mails:ya_khol@franko.lviv.ua, \\ olga.mylyo@gmail.com
}

\begin{abstract}
Let $\mathrm{sn}_{i} z$ be algebraically independent Jacobi elliptic functions, $\left(4 K_{i}, 2 i K_{i}^{\prime}\right)$ be the main periods and $\varkappa_{1}, \varkappa_{2}$ be algebraic moduli of $\operatorname{sn}_{i} z(i=1,2)$. We estimate from below the simultaneous approximation of $\mathrm{sn}_{1} 4 K_{2}, \mathrm{sn}_{2} 4 K_{1}$.
\end{abstract}

Key words: simultaneous approximation, Jacobi elliptic function.

\section{INTRODUCTION}

Jacobi's elliptic function $\mathrm{sn} z$ satisfies the equation $\left(\mathrm{sn}^{\prime} z\right)^{2}=\left(1-\mathrm{sn}^{2} z\right)\left(1-\varkappa^{2} \mathrm{sn}^{2} z\right)$ ([1]). The number $\varkappa$ is called the modul sn $z, 0<\varkappa<1$, the number $\varkappa^{\prime}=\left(1-\varkappa^{2}\right)^{1 / 2}$ is called its additional modulus. The pair of main periods of $\operatorname{sn} z$ is $\left(4 K, 2 i K^{\prime}\right)$, where $K, K^{\prime}$ are the complete elliptic integrals of the first kind that correspond to $\varkappa, \varkappa^{\prime}$ [1].

Denote by $\operatorname{sn}_{1} z, \mathrm{sn}_{2} z$ two algebraically independent Jacobi elliptic functions determined by algebraic $\varkappa_{1}, \varkappa_{2}$ respectively, $0<\varkappa_{1}<1,0<\varkappa_{2}<1 ;\left(4 K_{1}, 2 i K_{1}^{\prime}\right)$, $\left(4 K_{2}, 2 i K_{2}^{\prime}\right)$ are pairs of their main periods.

We denote by $d(P), L(P)$ the degree and the length of the polynomial $P$ with integers coefficients, by $d(\alpha), L(\alpha)$ the degree and length of the algebraic number $\alpha$ [2]; $\xi_{i}, i=1,2$, algebraic numbers, $n_{i}=d\left(\xi_{i}\right)$ and $L_{i}=L\left(\xi_{i}\right)$ their degrees and lengths respectively, $n=\operatorname{deg} \mathbb{Q}\left(\xi_{1}, \xi_{2}\right)$.

Theorem 1. Let

$$
T=n\left[\frac{\ln L_{1}}{n_{1}}+\frac{\ln L_{2}}{n_{2}}+\ln n\right] .
$$

2010 Mathematics Subject Classification: 11J82

(c) Kholyavka, Ya., Mylyo, O., 2019 
If there exists $C>0$ such that for all $k_{1}, k_{2} \in \mathbb{Z}, k_{1}^{2}+k_{2}^{2} \neq 0,\left|k_{1}\right|,\left|k_{2}\right|<t$, we have

$$
\left|k_{1} K_{1}+k_{2} K_{2}\right|>\exp \left(C t^{3}\right) \text {, }
$$

and at least one of the numbers $\mathrm{sn}_{1} 4 K_{2}, \mathrm{sn}_{2} 4 K_{1}$ is transcendent, then for arbitrary algebraic numbers $\xi_{1}, \xi_{2}$,

$$
\max \left\{\left|\operatorname{sn}_{1} 4 K_{2}-\xi_{1}\right|,\left|\operatorname{sn}_{2} 4 K_{1}-\xi_{2}\right|\right\}>\exp \left(-\Lambda T^{2} \ln T\right),
$$

where $\Lambda>0$ is a constant that depends only on $\varkappa_{1}, \varkappa_{2}$.

The approximation estimate formulated in Theorem 1 can be used, for example, to study the properties of elliptic Jacobi curves. Similar estimates for other numbers can be found in $[3]-[5]$.

\section{Auxiliary statements}

We formulate the basic lemmas, which are necessary to prove Theorem 1 . Let $c_{i}$, for all $i$, be positive constants, dependent only on $\varkappa_{1}$ and $\varkappa_{2}$.

Lemma 1. For each integer $m \geqslant 1$, there exist polynomials with the integer coefficients $P_{1, m}$ and $Q_{1, m}$ such that

$$
\operatorname{sn} m z=\frac{P_{1, m}\left(\operatorname{sn} z, \mathrm{sn}^{\prime} z\right)}{Q_{1, m}\left(\varkappa^{2}, \operatorname{sn} z\right)}
$$

where $L\left(P_{1, m}\right), L\left(Q_{1, m}\right) \leqslant \exp \left(c_{1} m^{2}\right), \operatorname{deg} P_{1, m}, \operatorname{deg} Q_{1, m} \leqslant m^{2}, i=1,2$.

Lemma 2. Let $m \in \mathbb{N}$. Then there are polynomials $P_{s, l} \in \mathbb{Z}\left[x_{1}, x_{2}, x_{3}\right]$ such that

$$
\left(\operatorname{sn}^{l} z\right)^{(s)}=\frac{d^{s}}{d w^{s}}\left((\operatorname{sn} z)^{l}\right)=P_{s, l}\left(\varkappa^{2}, \operatorname{sn} z, \operatorname{sn}^{\prime} z\right),
$$

$\operatorname{deg}_{x_{1}} P_{s, l} \leq s+l, \operatorname{deg}_{x_{2}} P_{s, l} \leq s+2 l, \operatorname{deg}_{x_{3}} P_{s, l} \leq 1, L\left(P_{s, l}\right) \leq \exp \left(c_{2} s \log (s+l)\right)$.

Proofs of Lemma 1 and Lemma 2 are similar to the proof of properties of the function $\wp(z)[8]$.

Lemma 3 ([1]). If $z, w, z+w$ are different from the poles $\operatorname{sn} z$, then

$$
\operatorname{sn}(z+w)=\frac{\operatorname{sn} z \operatorname{sn}^{\prime} w+\operatorname{sn} w \operatorname{sn}^{\prime} z}{1-\varkappa^{2} \operatorname{sn} z^{2} \operatorname{sn} w^{2}} .
$$

Lemma 4 ([6]). Let $\alpha, \beta$ be arbitrary algebraic numbers, $\gamma^{2}=\left(1-\alpha^{2}\right)\left(1-\alpha^{2} \beta^{2}\right)$. Then

$$
L(\gamma)<\exp \left(6 \operatorname{deg} \mathbb{Q}(\alpha, \beta)\left(\frac{\ln L(\alpha)}{d(\alpha)}+\frac{\ln L(\beta)}{d(\beta)}+1\right)\right), \quad d(\gamma) \geq \frac{\operatorname{deg} \mathbb{Q}(\alpha, \beta)}{\min (2 d(\alpha), 4 d(\beta))} .
$$

Lemma 5 ([4]). Let $B, P \in \mathbb{N}, Q_{p, b} \in \mathbb{Z}\left[x_{1}, \ldots, x_{n}\right], 0 \leq b<B, 0 \leq p<P, L\left(Q_{p, b}\right) \leq L$, $\operatorname{deg}_{x_{i}} Q_{p, b} \leq \mathcal{N}_{i} ; \alpha_{1}, \ldots, \alpha_{n}$ be algebraic numbers, $m=\operatorname{deg} \mathbb{Q}\left(\alpha_{1}, \ldots, \alpha_{n}\right)$. If $P>m B$, then the system of linear equations

$$
\sum_{p=0}^{P-1} x_{p} Q_{p, b}\left(\alpha_{1}, \ldots, \alpha_{n}\right)=0, \quad 0 \leq b<B
$$

has integer rational solutions $A_{0}, \ldots, A_{P-1}$ such that

$$
0<\max \left|A_{i}\right|<1+(L P)^{\frac{m B}{P-m B}}\left(\prod_{i=1}^{n}\left(1+\mathcal{N}_{i}\right)\left(L\left(\alpha_{i}\right)\left(1+d\left(\alpha_{i}\right)\right)\right)^{\frac{\mathcal{N}_{i}}{d\left(\alpha_{i}\right)}}\right)^{\frac{m B}{P-m B}} .
$$


Lemma 6 ([2]). Let $\alpha_{1}, \ldots, \alpha_{n}$ be algebraic numbers, $P \in \mathbb{Z}\left[x_{1}, \ldots, x_{n}\right], \operatorname{deg}_{x_{i}} P \leq \mathcal{N}_{i}$, $m=\operatorname{deg} \mathbb{Q}\left(\alpha_{1}, \ldots, \alpha_{n}\right)$. If $P\left(\alpha_{1}, \ldots, \alpha_{n}\right) \not \equiv 0$, then

$$
\left|P\left(\alpha_{1}, \ldots, \alpha_{n}\right)\right| \geq L(P)^{1-m} \prod_{i=1}^{n} L\left(\alpha_{i}\right)^{\frac{-\mathcal{N}_{i} m}{d\left(\alpha_{i}\right)}} .
$$

Denote by $|f(z)|_{D}=\sup _{|z| \leq D}|f(z)|$.

Lemma 7 ([5]). The functions $\sigma(z)$ and $\sigma(z-\omega) \operatorname{sn} z$ are integer and for $M>1$ estimates

$$
|\sigma(z-\omega) \operatorname{sn} z|_{M},|\sigma(z)|_{M} \leq C_{1}^{M^{2}}
$$

hold, $\omega$ is the corresponding half-life of the Weierstrass function and $\sigma(z)$ be a $\sigma$-function of Weierstrass which corresponds to the function $\wp(z)$ associated with $\operatorname{sn}(z)$.

If $\varepsilon$ is the distance from the nearest pole of $\operatorname{sn} z$ to $z_{0}$ and $\left|z_{0}\right| \leq M$, then $\left|\sigma\left(z_{0}\right)\right| \geq$ $\varepsilon C_{2}^{-M^{2}}$, where $C_{1}, C_{2}$ are constants dependent only on $\varkappa$.

Lemma 8 (the Hermite formula, [2]). Let $f(\zeta)$ be a regular function in the circle $\Gamma$ of radius $R, a_{1}, \ldots, a_{m} \in \Gamma, a_{i} \neq a_{j}$ if $i \neq j, s \in \mathbb{N}_{0}$. Then for an arbitrary inner point $z \in \Gamma$, other than $a_{1}, \ldots, a_{m}$, the equality

$$
\begin{gathered}
f(z)=\frac{1}{2 \pi i} \oint_{\partial \Gamma} \prod_{k=1}^{m}\left(\frac{z-a_{k}}{\zeta-a_{k}}\right)^{s+1} \frac{f(\zeta) d \zeta}{\zeta-z}- \\
-\frac{1}{2 \pi i} \sum_{i=1}^{m} \sum_{\tau=1}^{s} \frac{f^{(\tau)}\left(a_{i}\right)}{\tau !} \oint_{\left|\zeta-a_{i}\right|=\rho_{i}} \prod_{k=1}^{m}\left(\frac{z-a_{k}}{\zeta-a_{k}}\right)^{s+1} \frac{\left(\zeta-a_{i}\right)^{\tau}}{\zeta-z} d \zeta
\end{gathered}
$$

holds, where $\rho_{i}$ is enough small, $\left\{\zeta:\left|\zeta-a_{i}\right| \leq \rho_{i}\right\} \subset \Gamma$ and not contain points $z i$ $a_{k}, k \neq i$.

Lemma 9 ([1], [7]). Let $P \in \mathbb{C}\left[x_{1}, x_{2}\right], P\left(x_{1}, x_{2}\right) \not \equiv 0$, be a polynomial of degree at most $\mathcal{D}_{1}$ by $x_{1}$ and $\mathcal{D}_{2}$ by $x_{2}, \mathcal{D}_{1}, \mathcal{D}_{2} \geq 1, \mathrm{sn}_{1} z, \mathrm{sn}_{2} z$ be algebraically Jacobi independent elliptic functions. Then the number of zeros $P\left(\mathrm{sn}_{1} z, \mathrm{sn}_{2} z\right)$ given with their multiplicities at $|z|<K$ does not exceed $C_{3} K^{2}\left(\mathcal{D}_{1}+\mathcal{D}_{2}\right)$, where $C_{3}$ is some constant that is independent of the polynomial.

\section{Proof of Theorem 1}

We will prove Theorem 1 using the second Gelfond method described in [2], [3]. Suppose that (1) does not hold, that is, for sufficiently large $\lambda \in \mathbb{N}$,

$$
\max \left\{\left|\operatorname{sn}_{1} 4 K_{2}-\xi_{1}\right|,\left|\operatorname{sn}_{2} 4 K_{1}-\xi_{2}\right|\right\}<\exp \left(-\lambda^{7} T^{2} \ln T\right) .
$$

Let

$$
S=L=\lambda^{3} \ln \lambda T, N=\lambda \sqrt{\lambda T},
$$

$$
F(z)=\sum_{l_{1}=0}^{L} \sum_{l_{2}=0}^{L} C_{l_{1}, l_{2}} \operatorname{sn}_{1}^{l_{1}} z \mathrm{sn}_{2}^{l_{2}} z, C_{l_{1}, l_{2}}=\sum_{\tau=1}^{n} C_{l_{1}, l_{2}, \tau} \zeta_{\tau}, C_{l_{1}, l_{2}, \tau} \in \mathbb{Z},
$$

where $\zeta_{\tau}$ are the generic elements of $\mathbb{Q}\left(\xi_{1}, \xi_{2}\right)$. 
Denote by $\varphi_{i, 1}(z)=\operatorname{sn}_{i}\left(z+\frac{K_{i}}{2}\right), \varphi_{i, 2}(w)=\operatorname{sn}_{i}\left(w+\frac{3 K_{i}}{2}\right), i=1,2$. Then (Lemma 3)

$$
\operatorname{sn}_{i}(z+w)=\frac{\varphi_{i, 1}(z) \varphi_{i, 2}^{\prime}(w)+\varphi_{i, 2}(w) \varphi_{i, 1}^{\prime}(z)}{1-\varkappa_{i}^{2} \varphi_{i, 1}^{2}(z) \varphi_{i, 2}^{2}(w)}=\frac{\Lambda_{i, 1}(z, w)}{\Lambda_{i, 2}(z, w)} .
$$

Let

$$
G_{i, s, k, l}\left(\varkappa_{i}, z\right)=\left.\frac{d^{s}}{d w^{s}}\left(\Lambda_{i, 1}^{k}(z, w) \Lambda_{i, 2}^{l}(z, w)\right)\right|_{w=0}
$$

The so defined polynomials satisfy $\operatorname{deg} G_{i, s, k, l} \leq 4(k+l), \ln L\left(G_{i, s, k, l}\right) \leq s \ln (s(k+l)+$ $\left.c_{3}(s+k+l)\right)$.

With (3) just like in [7], [8], we get

$$
\begin{gathered}
F^{(s)}(z)=\left.\frac{d^{s}}{d w^{s}}\left(\left(\Lambda_{1,2}^{-L}(z, w) \Lambda_{2,2}^{-L}(z, w)\right)\left(F(z+w) \Lambda_{1,2}^{L}(z, w) \Lambda_{2,2}^{L}(z, w)\right)\right)\right|_{w=0}= \\
=\left.\sum_{t=0}^{s}\left(\begin{array}{l}
s \\
t
\end{array}\right) \frac{d^{s-t}}{d w^{s-t}}\left(\Lambda_{1,2}^{-L}(z, w) \Lambda_{2,2}^{-L}(z, w)\right)\right|_{w=0} F_{s, t}(z)
\end{gathered}
$$

where

$$
F_{s, t}(z)=\sum_{l_{1}=0}^{L} \sum_{l_{2}=0}^{L} C_{l_{1}, l_{2}} \sum_{i=0}^{t}\left(\begin{array}{l}
t \\
i
\end{array}\right) G_{1, t-i, l_{1}, L-l_{1}}\left(\varkappa_{1}, z\right) G_{2, i, l_{2}, L-l_{2}}\left(\varkappa_{2}, z\right) .
$$

Applying Lemma 4 for $\alpha=\xi_{1}, \beta=\varkappa_{1}, \gamma=\xi_{3}$, we get the estimate $d\left(\xi_{3}\right)$ and $L\left(\xi_{3}\right)$ of the number $\xi_{3}$, which approximates $\operatorname{sn}_{1}^{\prime} 4 K_{2}$, and in the case of $\alpha=\xi_{2}, \beta=\varkappa_{2}$, $\gamma=\xi_{4}$ we get the estimate $d\left(\xi_{4}\right)$ and $L\left(\xi_{4}\right)$ of the number $\xi_{4}$, which approximates $\mathrm{sn}_{2}^{\prime} 4 K_{1}$. Denote by $F_{s, n_{1}, n_{2}}\left(\xi_{1}, \ldots, \xi_{4}\right)$ and $F_{s, t, n_{1}, n_{2}}\left(\xi_{1}, \ldots, \xi_{4}\right)$ the expressions derived from $F^{(s)}\left(4 n_{1} K_{1}+4 n_{2} K_{2}\right)$ and $F_{s, t}\left(4 n_{1} T K_{1}+4 n_{2} K_{2}\right)$ by replacing $\operatorname{sn}_{1} 4 K_{2}, \mathrm{sn}_{2} 4 K_{1}$, $\mathrm{sn}_{1}^{\prime} K_{2}, \mathrm{sn}_{2}^{\prime} K_{1}$ by $\xi_{1}, \ldots, \xi_{4}$ respectively and apply Lemma 5 to $F_{s, t, n_{1}, n_{2}}\left(\xi_{1}, \ldots, \xi_{4}\right)$. We will consider $F_{s, t, n_{1}, n_{2}}\left(\xi_{1}, \ldots, \xi_{4}\right)$ for $1 \leq n_{1}, n_{2} \leq N, 0 \leq t \leq s \leq S$ as $N^{2} S$ linear forms of $n L^{2}$ variables $C_{l_{1}, l_{2}, \tau}$. Having used Lemmas $1-6$ and (3)-(7), we choose not all equal to zero $C_{l_{1}, l_{2}, \tau}$ such that for $1 \leq n_{1}, n_{2} \leq N, 0 \leq t \leq s \leq S$,

$$
\begin{gathered}
F_{s, t, n_{1}, n_{2}}\left(\xi_{1}, \ldots, \xi_{4}\right)=0, \\
\left|C_{l_{1}, l_{2}, \tau}\right|<\exp \left(c_{4} \lambda^{6} \ln \lambda T^{2} \ln T\right) .
\end{gathered}
$$

With (4), (3), (9) and Lemmas $1-5$ we get for $1 \leq n_{1}, n_{2} \leq N, 0 \leq s \leq S$,

$$
\left|F^{(s)}\left(4 n_{1} K_{1}+4 n_{2} K_{2}\right)-F_{s, n_{1}, n_{2}}\left(\xi_{1}, \ldots, \xi_{4}\right)\right|<\exp \left(-\frac{1}{4} \lambda^{7} T^{2} \ln T\right) .
$$

From (8), (10) at $1 \leq n_{1}, n_{2} \leq N, 0 \leq s \leq S$ we get

$$
\left|F^{(s)}\left(4 n_{1} K_{1}+4 n_{2} K_{2}\right)\right|<\exp \left(-\frac{1}{4} \lambda^{7} T^{2} \ln T\right) .
$$

We show that (11) also holds for $1 \leq n_{1}, n_{2} \leq N, 0 \leq s \leq \lambda S$. [1],

Let $\wp_{i}(u)$ and $\sigma_{i}(u)$ correspond to the functions $\operatorname{sn}_{i} z, i=1,2, u=z\left(e_{1, i}-e_{3, i}\right)^{-1 / 2}$,

$$
G(z)=F(z) \sigma_{1}^{L}\left(u+\omega_{3,1}\right) \sigma_{2}^{L}\left(u+\omega_{3,2}\right),
$$

where $\omega_{j, i}$ is the half-period of $\wp_{i}(u)$. Choose the least possible integer $r$ such that

$$
r>32(N+1)\left(\left|K_{1}\right|+\left|K_{2}\right|+\left|K_{1}^{\prime}\right|+\left|K_{2}^{\prime}\right|\right) .
$$


Denote by $R=48 r$. Then with Lemmas $1-5$, Lemma 8 and (1), (3), (3), (9), (12),

$$
|G(z)|_{R}<\exp \left(-\lambda^{6} \ln \lambda T^{2} \ln T\right) .
$$

From (13) we get for $0 \leq s \leq \lambda S$

$$
\left|G^{(s)}(z)\right|_{r}<\exp \left(-\frac{1}{2} \lambda^{6} \ln \lambda T^{2} \ln T\right) .
$$

For a sufficiently small $\varepsilon$ in the $\varepsilon$-neighborhood of the points $4 n_{1} K_{1}$ the function $\sigma_{2}\left(z-\omega_{2}\right)$ and in the $\varepsilon$-neighborhood of the points $4 n_{2} K_{2}$ the function $\sigma_{1}\left(z-\omega_{1}\right)$ has no zeros, so from (2) and Lemma 7 for $n_{1}, n_{2} \leq 32 N$ we obtain

$$
\left|\sigma_{i}\left(z-\omega_{i}\right)\right|_{z \in V\left(\varepsilon, 4 n_{1} K_{1}+4 n_{2} K_{2}\right)}>\exp \left(-c_{5} \lambda^{5} \ln \lambda T^{2}\right) .
$$

From (13)-(15) for $1 \leq n_{1}, n_{2} \leq N, 0 \leq s \leq \lambda S$ we get

$$
\left|F^{(s)}\left(4 n_{1} K_{1}+4 n_{2} K_{2}\right)\right|<\exp \left(-\frac{\lambda^{6}}{3} \ln \lambda T^{2} \ln T\right) .
$$

Given (10), for $1 \leq n_{1}, n_{2} \leq N$ and $0 \leq s \leq \lambda S$, from (16) we obtain

$$
\left|F_{s, n_{1}, n_{2}}\left(\xi_{1}, \ldots, \xi_{4}\right)\right|<\exp \left(-\frac{\lambda^{6}}{4} \ln \lambda T^{2} \ln T\right) .
$$

Considering $F_{s, t, n_{1}, n_{2}}\left(\xi_{1}, \ldots, \xi_{4}\right)$ as a value of the corresponding polynomial in algebraic points, from lemma 6 , (1), (3) we get for $0 \leq t \leq s \leq \lambda S, 1 \leq n_{1}, n_{2} \leq N$, $F_{s, t, n_{1}, n_{2}}\left(\xi_{1}, \ldots, \xi_{4}\right) \neq 0$, we obtain the estimate

$$
\left|F_{s, t, n_{1}, n_{2}}\left(\xi_{1}, \ldots, \xi_{4}\right)\right|>\exp \left(-\lambda^{5} \ln \lambda T^{2} \ln T\right) .
$$

From (3), (18) we get for $0 \leq s \leq \lambda S, 1 \leq n_{1}, n_{2} \leq N$

$$
\left|F_{s, n_{1}, n_{2}}\left(\xi_{1}, \ldots, \xi_{4}\right)\right|>\exp \left(-2 \lambda^{5} \ln \lambda T^{2} \ln T\right) .
$$

Since (17) and (19) are conflicting, we get for $1 \leq n_{1}, n_{2} \leq N, 0 \leq s \leq \lambda S$,

$$
F_{s, n_{1}, n_{2}}\left(\xi_{1}, \ldots, \xi_{4}\right)=0 .
$$

From (20) it follows that the polynomial $F(z)$ has at least $c_{6} \lambda^{7} \ln \lambda T^{2}$ zeros (with multiplicities). From Lemma 9 we get that the number of zeros may not be more than $c_{7} \lambda^{6} \ln \lambda T^{2}$, so for sufficiently large $\lambda$ assumption (4) leads to a contradiction, which proves the theorem.

\section{REFERENCES}

1. D. F. Lawden, Elliptic functions and applications, Springer, Berlin, 1989.

2. N. I. Fel'dman, Hilbert's seventh problem, Moskov State Univ., Moskov, 1982 (in Russian).

3. N. I. Fel'dman and Yu. V. Nesterenko, Transcendental numbers, Springer, Berlin, 1998.

4. E. Reyssat, Approximation algébrique de nombres lies aux fonctions elliptiques et exponentielle, Bull. Soc. Math. Fr. 108 (1980), 47-79. DOI: 10.24033/bsmf.1908

5. D. Masser, Elliptic functions and transcendence, Springer, Berlin, 1975.

6. Ya. M. Kholyavka, On the simultaneous approximation of invariants of the elliptic function by algebraic numbers, Diophantine Analysis, Mosk. Univ. Press, Moscow, 1986, Part 2, 114-121 (in Russian)

7. Ю. В. Нестеренко, О мере алгебрачческой независимости значений эллиптической функиии, Изв. РАН. Сер. матем. 59 (1995), nо. 4, 155-178; English version: Yu. V. Nesterenko, On a measure of algebraic independence of values of an elliptic function, Izv. Math. 59 (1995), no. 4, 815-838. DOI: 10.1070/IM1995v059n04ABEH000035 
8. G. V. Chudnovsky, Algebraic independence of the values of elliptic functions at algebraic points; Elliptic analogue of the Lindemann-Weierschtrass theorem, Invent. Math. 61 (1980), 267-290. DOI: 10.1007/BF01390068

Стаття: надійшла до редколегї 11.04.2019

прийнята до друку 03.02.2020

\title{
СУМІСНІ НАБЛИЖЕННЯ ЗНАЧЕНЬ ЕЛІПТИЧНИХ ФУНКЦІЙ ЯКОБІ В ЇХНІХ ДІЙСНИХ ПЕРІОДАХ
}

\section{Ярослав ХОЛЯВКА, Ольга МИЛЬО}

\author{
Лъвівсъкий націоналъний університет імені Івана Франка, \\ вул. Університетсъка, 1, 79000, Львів \\ e-mails:ya_khol@franko.lviv.ua, \\ olga.mylyo@gmail.com
}

Нехай $\operatorname{sn}_{1} z, \operatorname{sn}_{2} z$ - алгебрично незалежні еліптичні функції Якобі. Позначимо через $\varkappa_{1}, 0<\varkappa_{1}<1$, еліптичний модуль $\operatorname{sn}_{1} z$, а через $\varkappa_{2}, 0<\varkappa_{2}<$ $1,-$ еліптичний модуль $\operatorname{sn}_{2} z$. Будемо припускати, що $\varkappa_{1}$ та $\varkappa_{2}$ алгебричні числа. Також позначимо через $\left(4 K_{1}, 2 i K_{1}^{\prime}\right)$ пару основних періодів $\operatorname{sn}_{1} z$, а через через $\left(4 K_{2}, 2 i K_{2}^{\prime}\right)$ - пару основних періодів $\mathrm{sn}_{2} z$, де $K_{1}, K_{2}, K_{1}^{\prime}$ та $K_{2}^{\prime}$ є дійсними числами.

Еліптична функція Якобі $\mathrm{sn}_{1} z$ задовольняє рівняння

$$
\left(\mathrm{sn}_{1}^{\prime} z\right)^{2}=\left(1-\mathrm{sn}_{1}^{2} z\right)\left(1-\varkappa_{1}^{2} \operatorname{sn}_{1}^{2} z\right),
$$

а еліптична функція Якобі $\mathrm{sn}_{2} z$ задовольняе рівняння

$$
\left(\operatorname{sn}_{2}^{\prime} z\right)^{2}=\left(1-\operatorname{sn}_{2}^{2} z\right)\left(1-\varkappa_{2}^{2} \operatorname{sn}_{2}^{2} z\right) .
$$

Числа $\varkappa_{1}^{\prime}=\left(1-\varkappa_{1}^{2}\right)^{1 / 2}$ та $\varkappa_{2}^{\prime}=\left(1-\varkappa_{2}^{2}\right)^{1 / 2}$ називають додатковими еліптичними модулями еліптичних функцій Якобі $\operatorname{sn}_{1} z$ i $\mathrm{sn}_{2} z$. Числа $K_{1}, K_{2}, K_{1}^{\prime}$ та $K_{2}^{\prime} є$ повні еліптичні інтеграли першого роду, що відповідають числам

$$
\begin{gathered}
\varkappa_{1}, \varkappa_{2}, \varkappa_{1}^{\prime} \text { та } \varkappa_{2}^{\prime} \text {. Покладемо } I(v)=\int_{0}^{\pi / 2}\left(1-v^{2} \sin ^{2} t\right)^{-1 / 2} d t . \text { Тоді } \\
K_{1}=I\left(\varkappa_{1}\right), K_{2}=I\left(\varkappa_{2}\right), K_{1}^{\prime}=I\left(\varkappa_{1}^{\prime}\right) \text { та } K_{2}^{\prime}=I\left(\varkappa_{2}^{\prime}\right) .
\end{gathered}
$$

Число $а$ називають алгебричним числом, якщо існує такий многочлен $P(x)$ 3 цілими коефіцієнтами, що $P(a)=0$. Многочлен $P(x) \in \mathbb{Z}[x]$ назвемо основним многочленом для числа $a$, якщо він задовольняе такі умови: $P(a)=0$; старший коефіцієнт $P(x)$ додатний; коефіцієнти $P(x)$ взаємно прості цілі числа; $P(x)$ незві дний над $\mathbb{Q}$. Довжиною $P(x)$ назвемо суму модулів його коефіцієнтів. Степенем числа а називають степінь його основного многочлена $P(x)$ і позначають $\operatorname{deg} a$, довжиною числа а називають довжину його основного многочлена $P(x)$ і позначають $L(a)$.

Нехай $\xi_{1}, \xi_{2}-$ довільні алгебричні числа, $n_{1}=\operatorname{deg}\left(\xi_{1}\right), n_{2}=\operatorname{deg}\left(\xi_{2}\right)$, 
$L_{1}=L\left(\xi_{1}\right), L_{2}=L\left(\xi_{2}\right)$ та $n=\operatorname{deg} \mathbb{Q}\left(\xi_{1}, \xi_{2}\right)$.

У статті отримано оцінку сумісного наближення чисел $\operatorname{sn}_{1} 4 K_{2}$ та $\mathrm{sn}_{2} 4 K_{1}$.

Теорема 1. Нехай

$$
T=n\left[\frac{\ln L_{1}}{n_{1}}+\frac{\ln L_{2}}{n_{2}}+\ln n\right.
$$

Якщо існуе така постійна $C>0$, що для всіх $k_{1}, k_{2} \in \mathbb{Z}, k_{1}^{2}+k_{2}^{2} \neq 0$, $\left|k_{1},\right| k_{2} \mid<t$, справджуетъся

$$
\left|k_{1} K_{1}+k_{2} K_{2}\right|>\exp \left(C t^{3}\right),
$$

та хоча б одне з чисел $\mathrm{sn}_{1} 4 K_{2}, \mathrm{sn}_{2} 4 K_{1}$ е трансцендентним, то для довілъних алгебричних чисел $\xi_{1}, \xi_{2}$, справджуєтъсл оцінка

$$
\max \left\{\left|\operatorname{sn}_{1} 4 K_{2}-\xi_{1}\right|,\left|\operatorname{sn}_{2} 4 K_{1}-\xi_{2}\right|\right\}>\exp \left(-\Lambda T^{2} \ln T\right),
$$

де $\Lambda>0$ е константа, залежна тільки від $\varkappa_{1}, \varkappa_{2}$.

Зауважимо, що числа, кратні $K_{2}$, не є полюсами $\operatorname{sn}_{1} z$, а числа, кратні $K_{1}$, не є полюсами $\mathrm{sn}_{2} z$.

Оцінки наближень, подібні до отриманої в теоремі 1, можна використовувати для вивчення арифметичних властивостей еліптичних кривих Якобі. Доведення теореми 1 проводиться за допомогою другого методу Гельфонда. Припускаємо, що для достатньо великого $\lambda \in \mathbb{N}$, виконується

$$
\max \left\{\left|\operatorname{sn}_{1} 4 K_{2}-\xi_{1}\right|,\left|\operatorname{sn}_{2} 4 K_{1}-\xi_{2}\right|\right\}<\exp \left(-\lambda^{7} T^{2} \ln T\right)
$$

і покажемо, що це припущення приводить до протиріччя.

При доведенні вибираємо такі значення параметрів

$$
S=L=\lambda^{3} \ln \lambda T, N=\lambda \sqrt{\lambda T},
$$

та допоміжну функцію

$$
F(z)=\sum_{l_{1}=0}^{L} \sum_{l_{2}=0}^{L} C_{l_{1}, l_{2}} \operatorname{sn}_{1}^{l_{1}} z \operatorname{sn}_{2}^{l_{2}} z, C_{l_{1}, l_{2}}=\sum_{\tau=1}^{n} C_{l_{1}, l_{2}, \tau} \zeta_{\tau}, C_{l_{1}, l_{2}, \tau} \in \mathbb{Z},
$$

де $\zeta_{\tau}$ є твірними елементами $\mathbb{Q}\left(\xi_{1}, \xi_{2}\right)$.

Ключові слова: сумісні наближення, еліптична функція Якобі. 